\title{
Analysis on Development and Application of Power Electric Device and Variable Frequency Technology
}

\author{
Hongyin Bao* \\ Haiwei New Energy Co., Ltd. of CSIC, Urumqi, Xinjiang 830000, China
}

\begin{abstract}
Today, with rapid development of science and technology in the 21 st century, China has also obtained great achievements drawing world's attention regarding application and research in power electronics technology and variable frequency technology field. This paper has intensively studied and discussed development and application of power electronic device and variable frequency technology. This paper has first analyzed current application situation and trends of power electronic device in new energy and power system, rail transit and electric car, energy saving of industrial motor, consumption electronics, and national defense and war industry, then discussed development history of variable frequency technology, hazards brought by power electronic device and countermeasures, and finally demonstrated future development of power electronics technology and expected a broad development prospect of variable-frequency regulating speed technology in the future.
\end{abstract}

\author{
KEYWORDS \\ Electronic device \\ Variable frequency technology \\ Harmonic wave \\ Interference suppression
}

\section{Introduction}

Demands of modern industrial production and social development promote rapid development of AC-AC variable frequency technology. Development and application of modern power electronic device, modern control theory and control device, and microprocessor control technology and large-scale integrated circuit have created material and technical conditions for development and application of AC variable frequency technique. A full digital control technique with micro-processing technique, PWM control chip and power electronic switch device as well as sinusoidal pulse width modulation (SPWM) technique, vector transfer technique, direct torque control and network communication technique is used as control approach. AC variable frequency technology has experienced a process from traditional AC-AC direct variable frequency to AC$\mathrm{DC}-\mathrm{AC}$ variable frequency and then matrix $\mathrm{AC}-\mathrm{AC}$ variable frequency.

Copyright $\odot 2015$ Hongyin Bao

doi: $10.18686 /$ esta.v2i1.7

Received: October 5, 2015; Accepted: October 29, 2015; Published online: December 2

This is an open-access article distributed under the terms of the Creative Commons Attribution Unported License (http://creativecommons.org/ licenses/by-nc/4.0/), which permits unrestricted use, distribution, and reproduction in any medium, provided the original work is properly cited.

${ }^{*}$ Corresponding author: Haiwei New Energy Co., Ltd. Of CSIC, Urumqi, Xinjiang 830000, China. E-mail: bhy1865@173.com

\section{Current Situation and Trends of Application of Power Electronic Device}

\subsection{Application in New Energy and Power System}

Power system is the most important and potential market field in the application of power electronics technology. The power electronics technology is widely and significantly applied in the whole process of generation, transmission, distribution and use of electric energy. If viewed from the perspective of power utilization, power electronics technology shall be utilized to conduct energy-saving technical reconstruction and improve power utilization efficiency; if viewed from the perspective of power generation, transmission and distribution, power electronics technology must be utilized to improve power generation efficiency and better power transmission and distribution quality.

\subsection{Application in Rail Transit and Electric Car}

Application of power electronics technology in rail transit traction system mainly includes three aspects, namely, main transmission system, auxiliary transmission system, and voltage-stabilized source in control and auxiliary system. With the promotion of power electronics technology, electric transmission system is directly developed from DC transmission to modern AC transmission. Improvement of capacity and performance of power electronic device, bettering of encapsulation form, and employment of modularized design technique of functional unit have promoted 
simplification of traditional system device and accelerated development of traction electric transmission system, auxiliary system, and control and auxiliary current voltagestabilized source [1].

\subsection{Application in Industrial Motor Energy-saving}

As the largest consumption carrier of electric energy, motor presents great electricity-saving potential. " $10^{\text {th }}$ Fiveyear" and " $11^{\text {th }}$ Five-year" planning of China included energy-saving of motor system as key energy-saving project. Besides, with quick development of power electronics technology, computer technology and automatic control technology, electric transmission technology is facing a history reform. To be specific, AC speed regulation is used to replace DC speed regulation, and computer digital control technology is used to replace analog control.

\subsection{Application in Consumption Electronics}

Application of power electronic technology in consumption electronics mainly focuses on motor driving, induction heating and lighting driving in various household appliances as well as power management of various personal electronic products. Household appliances rely on variable frequency technology and mainly aim at high function and power saving.

\subsection{Application in National Defense and Military Project}

Power electronics technology and power electronic device have been already extensively applied and penetrated in many important fields day by day, such as energy, environment, manufacturing and transportation especially advanced energy technology, laser technology, aerospace technology, high-grade CNC machine tool and basic manufacturing technology related to national security and national defense. Power electronics technology is a core technology related to the abovementioned fields. Power electronics is increasingly widely applied in modern national defense. Power electronics core technology is involved in special supply source, power driving, impelling and controlling of modern national defense equipment.

\section{Developing History of Variable Frequency Tech- nology}

Currently, variable frequency technology develops rapidly. From the very beginning, it could only change frequency but could not change voltage. Later, PWM variable voltage variable frequency control drawing wide attention from people was successfully developed. In the 1980s, the issue of PWM mode optimization rose people's desire to explore and research again. As a result, optimized modes such as phase shift carrier PWM technology, modulating wave longitudinal splitting method and in-phase carrier PWM were derived. VVVF frequency changer generally applied in each field of industry features good mechanical char- acteristics and hardness and easy control. Requirement of general transmission for smooth speed regulating can be fulfilled. However, the shortcoming of this control method is that it can result in reduction of output maximum torque during low-frequency operation. Vector control VVVF is realized through equivalent conversion of stator AC current of asynchronous motor under three-phase coordinate system by three phases and two phases to DC current under synchronous rotation coordinate system. The control of asynchronous motor is realized through control of DC motor. Direct torque control simplifies each troublesome step. It is a mathematical model analyzing AC motor under stator coordinate system, and control is implemented through flux linkage and torque of motor [2].

All VVVF variable frequency, vector control variable frequency and direct torque control variable frequency have various disadvantages such as big harmonic current, low input power factor and demand for big energy storage capacitor. Therefore, matrix-type AC-AC variable frequency is favored by people, for it can solve such disadvantages.

\section{Hazards Brought by Power Electronic Device and Countermeasures}

\subsection{Countermeasures for Harmonic Suppression}

Due to big conversion power, the switching frequency of high-voltage frequency changer is usually relatively small. Therefore, the output harmonic component ratio is relatively big. If regular sinusoidal pulse width modulation (SPWM) and space vector pulse width modulation (SVPWM) are adopted, the problem of big harmonic wave cannot be solved. Output voltage harmonic would affect motor operation to a certain extent, to result in motor torque pulse and increase of extra loss. Input harmonic would affect power grid. In recent years, some different rectification and inversion schemes have been put forward in aspects such as circuit structure and control technology by centering on effective inhibition and reduction of harmonic current and realization of more stable operation of motor and reduction of loss. As a result, a diversified bigpower variable frequency technology has been formed. In current stage, multi-pulse rectification, transformer coupling output and multi-level and unit cascade techniques are generally adopted, to form a big-power frequency changer main circuit with multi-pulse rectification topology or multi-level topology as input stage and transformer coupling output or multi-level output topology as output stage. For example, unit series multi-level technology adopted by Robicon of the United States and Harvest of China adopted multi-group low-voltage and small-power IGBT and PWM variable frequency unit series output to realize high-voltage big-power integration. Since power unit is adopted for series connection, problems of dynamic and static violating-sharing between elements would not occur as shown in Figure 1. Each variable frequency unit is power supplied by an isolation transformer with multiple 
windings, thus technically solving harmonic problem generated by general 6-pulse and 12-pulse frequency changer to realize perfect inharmonic frequency variation. Besides, some new pulse width modulation technique is also being continuously developed and studied. For example, Zhao Zhengming, et al. put forward a mixed PWM method. To be specific, SVPWM is adopted under low frequency and selective harmonic elimination pulse width modulation (SHEPWM) is adopted under high frequency, thus avoiding deterioration of harmonic characteristic of SVPWM under high frequency and big storage amount of SHEPWM under low frequency. Also, the advantages of the two are brought into full play so as to effectively inhibit loworder harmonics within the whole working range to obtain relatively favorable output waveform [3].

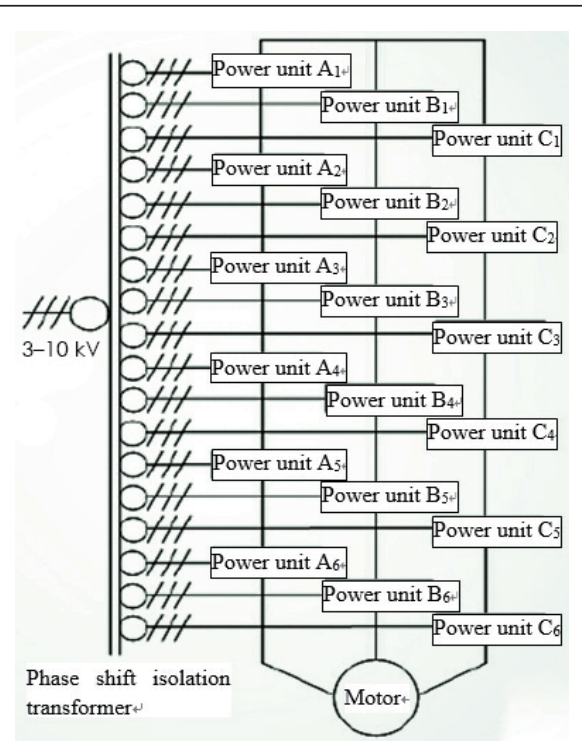

Figure 1. Serial Multi-level Inversion of Power Unit.

\subsection{Inhibition of Electromagnetic Interference}

Solution to EMI is overcome conduct conduction of switching device and occurrence of excessive current rise rate $\mathrm{di} / \mathrm{dt}$ and voltage rise rate $\mathrm{du} / \mathrm{dt}$ during disconnection. In current stage, zero current switch (ZCS) and zero voltage switch (ZVS) circuits draw much attention from people. The method is shown as follows:

(1) Serially connect inductance on switching device, which can inhibit di/dt upon conduction of switching device and avoid existence of voltage and current overlap in the device to effectively reduce positive closing loss;

(2) Parallel capacitance on switching device inhibits rise of du/dt after conduction of device and there is no voltage or current overlap on the device, so as to reduce switching loss;

(3) During conduction of anti-parallel diode on device, the switching device presents a zero voltage or zero current status. At this point, conduction or disconnection of driving device can realize actions of ZVS and ZCS.

Currently, partial resonance PWM and loss-free buffer circuit are software development technologies commonly applied.

\subsection{Power Factor Compensation}

The earliest method is to conduct power factor compensation through synchronous compensator. However, it generates very big noise and losses, the response speed is relatively slow, and the operation and maintenance are also complicated. Therefore, it cannot adapt to the actual situation and therefore it is gradually abandoned. The later method is to use static reactive compensation device of saturated resistor and it has overcome some disadvantages of synchronous compensator. However, it still fails to change the deadly features of big noise and losses. Besides, problems such as nonlinear circuit and failure of split phase to compensate imbalance of load also exist. Therefore, this method still cannot lead the trends. There is another method, i.e. static reactive generator. It boasts small volume, low cost, quick regulating speed and wide running range. Besides, it can greatly reduce the harmonic content in compensating current, which quickly makes it a representative of development direction of dynamic reactive compensation device.

\section{Future Development of Power Electronics Technology}

The world development relies on power electronics technology. The future prospect and direction of power electronics technology are extremely broad and its development is mainly reflected in the following aspects:

(1) Further study and apply materials to improve power, frequency and temperature range of devices and lower prices of devices.

(2) System integration: Acquire higher integration and improve reliability.

(3) Application of multi-level inverter in big-power inverter.

(4) Flexible AC power transmission technology is a power transmission technology implementing flexible and quick regulation of impedance, voltage and phase position of AC power transmission system based on power electronics technology and modern control technology. It can realize flexible control of AC power transmission power, significantly improve stable level of power system, and improve power supply quality of power system. Therefore, the flexible AC power transmission technology will realize increasingly extensive application.

(5) Improvement of intelligent power module of power electronics technology.

(6) Design of full semiconductor conversion system featuring small volume, low power consumption, light weight and freedom from power.

(7) R\&D and manufacturing of energy-saving and environment-friendly electric car: Design more efficient and useful household appliance products. We can see from above that the highlights of development application of 
equipment and system include frequency control speed regulation, smart power grid, car electronics, information and office automation, household special power supply, flexible AC power transmission, new energy, solar energy, wind energy and fuel power supply.

\section{Conclusions}

With rapid development of science and technology, China has also obtained achievements drawing world's attention regarding application and research in power electronics technology and variable frequency technology field. Research and discussion of development and application of power electronic device and variable frequency technology indicate: Currently, variable frequency technology is widely applied for motor dragging relying on advantages such as high efficiency, wide speed regulating range, high accuracy and good smoothness, especially for air-conditioning system and fan and pump loads in water supply system where favorable energy-saving effect can be obtained. The constant torque load of elevator can ensure comfort of elevator and safe and reliable flat bed. Therefore, it can be estimated that the future variable-frequency regulating speed technology will have a broad developing prospect.

\section{References}

1. Chen, Y., Ye, W. T., \& Zhu, Y. H. (2008). Research on Development and Application of Power Electronic Device and Variable Frequency Technology. Modern Science, 18, 87-88.

2. Huang, S. X. (2009). Research on Electronic Variable Frequency Technology. Silicon Valley, 23, 25.

3. Zhang, H. F. (2009). Discussion of Development and Application of Power Electronic Device and Variable Frequency Technology. Manager's Journal, 22, 383. 